\title{
Produção de Hidrogênio e Negro de Carbono a partir da Degradação de Metano por Plasma Térmico
}

\section{Hydrogen and Carbon Black Production from the Degradation of Methane by Thermal Plasma}

\author{
Leila Cottet*1; Vanessa Zanon Baldissarelli²; Luís Otávio de Brito Benetoli³; Nito \\ Angelo Debacher ${ }^{4}$
}

Resumo

O gás metano $\left(\mathrm{CH}_{4}\right)$ é um dos principais gases indutores do efeito estufa. Pesquisas científicas recentes visam minimizar o acúmulo deste gás na atmosfera e desenvolver processos capazes de produzir materiais estáveis com valor agregado. O plasma térmico é uma alternativa promissora para alcançar tais objetivos, pois possibilita a obtenção de $\mathrm{H}_{2}$ e carbono sólido a partir do $\mathrm{CH}_{4}$, sem a formação paralela de subprodutos como $\mathrm{CO}_{2}$ e $\mathrm{NO}_{\mathrm{x}}$. Neste trabalho, $\mathrm{o} \mathrm{CH}_{4}$ foi degradado por plasma térmico visando a produção de hidrogênio $\left(\mathrm{H}_{2}\right)$ e negro de carbono. A eficiência de degradação do $\mathrm{CH}_{4}$, o rendimento do processo e a caracterização do negro de carbono produzido foram estudados. Os melhores resultados obtidos foram no fluxo de $5 \mathrm{~L} \mathrm{~min}^{-1}$ de $\mathrm{CH}_{4}$ a porcentagem de degradação e a seletividade para a produção de $\mathrm{H}_{2}$ chegam a $98,8 \%$ e $48,4 \%$, respectivamente. Em fluxos menores que $5 \mathrm{~L} \mathrm{~min}^{-1}$, o rendimento na produção de $\mathrm{H}_{2}$ aumenta e chega a $91,9 \%$. O negro de carbono obtido apresenta estrutura amorfa, características hidrofóbicas e pode ser comercializado como material para compósitos, podendo ainda ser ativado química e/ou fisicamente e utilizado como material adsorvente.

Palavras-chave: Plasma térmico. Metano. Hidrogênio. Negro de carbono.

\begin{abstract}
Methane gas $\left(\mathrm{CH}_{4}\right)$ is the main inducer of the so called greenhouse gases effect. Recent scientific research aims to minimize the accumulation of this gas in the atmosphere and to develop processes capable of producing stable materials with added value. Thermal plasma technology is a promising alternative to these applications, since it allows obtaining $\mathrm{H}_{2}$ and solid carbon from $\mathrm{CH}_{4}$, without the parallel formation of byproducts such as $\mathrm{CO}_{2}$ and $\mathrm{NO}_{x}$. In this work, $\mathrm{CH}_{4}$ was degraded by thermal plasma in order to produce hydrogen $\left(\mathrm{H}_{2}\right)$ and carbon black. The degradation efficiency of $\mathrm{CH}_{4}$, selectivity for $\mathrm{H}_{2}$ production as well as the characterization of carbon black were studied. The best results were obtained in the $\mathrm{CH}_{4}$ flow rate of $5 \mathrm{~L} \mathrm{~min}^{-1}$ the degradation percentage and the selectivity for $\mathrm{H}_{2}$ production reached $98.8 \%$ and $48.4 \%$, respectively. At flow rates of less than $5 \mathrm{~L} \mathrm{~min}^{-1}$ the selectivity for $\mathrm{H}_{2}$ production increases and reaches $91.9 \%$. The carbon black has obtained amorphous with hydrophobic characteristics and can be marketed to be used in composite material, and can also be activated chemically and/or physically and used as adsorbent material.
\end{abstract}

Keywords: Thermal Plasma. Methane. Hydrogen. Carbon black.

Doutoranda em Química, Universidade Federal de Santa Catarina, leilacottet@hormail.com

2 Professora substituta do Departamento de Química, Universidade Federal de Santa Catarina, vanessabaldissarelli@gmail.com

3 Professor do Departamento de Química, Universidade Federal de Santa Catarina, luskywalcker@yahoo.com.br

4 Professor do Departamento de Química, Universidade Federal de Santa Catarina, nito.debacher@ufsc.br 


\section{Introdução}

Um dos problemas recentes enfrentados pela humanidade é a poluição do meio ambiente gerada principalmente pela atividade antropogênica e industrial. A queima de combustíveis fósseis (petróleo, carvão, gás natural) e a grande produção de resíduos, é uma das principais causas do aumento do dióxido de carbono $\left(\mathrm{CO}_{2}\right)$ e metano $\left(\mathrm{CH}_{4}\right)$ na atmosfera terrestre, sendo estes os principais gases precursores do efeito estufa (FARRELL; CULLING; LEIFER, 2013).

Pesquisas estão sendo realizadas, focadas principalmente em encontrar uma tecnologia que possibilite a reforma desses gases de uma maneira efetiva e não agressiva ao meio ambiente (REDDY; KIM; SONG, 2013). Entre a variedade de métodos, a tecnologia de plasma térmico tornou-se popular industrialmente, devido à sua elevada eficiência e por ser considerado um processo ambientalmente limpo (KIM et al., 2005), (GUO; KIM, 2008).

O plasma é um gás ionizado, formado por uma mistura de íons, elétrons, espécies neutras, fótons e espécies excitadas geradas por ionização através de descargas elétricas entre dois eletrodos (BOULOS; FAUCHAIS; PFENDER, 1994). A principal diferença entre um gás e o plasma é o fato do plasma ser condutor de eletricidade devido à presença de cargas elétricas livres (elétrons e íons) entre seus constituintes, que fazem o plasma ser eletricamente condutor e responder fortemente a campos eletromagnéticos (FRIDMAN, 2008). No plasma térmico, as espécies quimicamente ativas e altamente energéticas estão em equilíbrio termodinâmico com o gás circundante (STAACK, 2008), (EMMERT et al., 2013). Existem inúmeros exemplos de aplicações da tecnologia de plasma, entre eles: a degradação de gases, reações de síntese, modificações e tratamento de superfícies, produção de nanoestruturas, entre outros (CUBAS et al., 2005) (SAMAL; PARK, 2012), (BENETOLI et al., 2012), (XU et al., 2013). Dessas tecnologias, a de maior interesse no presente trabalho é a pirólise a plasma, uma vez que permite a degradação de substâncias indesejáveis, com produção de materiais com valor agregado.

No caso específico da pirólise do $\mathrm{CH}_{4}$ por plasma térmico, entre os produtos formados, destaca-se o hidrogênio, que pode ser utilizado como combustível, e o negro de carbono, utilizado como material de reforço e estabilidade térmica em plásticos, cimento, pneus, borrachas, etc. (XU et al., 2013), (YAN et al., 2012).

Processos convencionais de produção de hidrogênio e negro de carbono baseiam-se na combustão incompleta de hidrocarbonetos, sujeitos a baixos rendimentos e emissão de elevados níveis de poluentes. Devido à combustão incompleta na presença de oxigênio, o processo é inevitavelmente acompanhado pela emissão de $\mathrm{CO}, \mathrm{CO}_{2}, \mathrm{SO}_{2}$, $\mathrm{NO}_{\mathrm{x}}$ e hidrocarbonetos voláteis (KIM et al., 2005; FABRY; FLAMANT; FULCHERI, 2001). Além disso, a temperatura máxima de reação alcançável é de $1500 \mathrm{~K}$ a $2000 \mathrm{~K}$ (KIM et al., 2005).

Por outro lado, o processo de produção de negro de carbono e hidrogênio por plasma térmico, apesar de usar energia elétrica e um gás inerte como o argônio no processo apresenta diversas vantagens em sua aplicação que são compensatórias, como por exemplo: aumento da eficiência, redução do tempo de reação, baixo custo do equipamento, favorecendo e viabilizando sua aplicação industrial (CHEN et al., 2008), (CHAUBEY et al., 2013). O uso do argônio como gás plasmogênico evita a formação de $\mathrm{CO}_{2}$ no processo e é necessário apenas para iniciar a formação do plasma podendo ser substituído pelo próprio gás a ser degradado. Outro fator importante é que o processo a plasma possibilita a utilização de diferentes fontes de matéria prima, além do gás metano ou butano, como qualquer fonte de hidrocarbonetos ou gases provenientes de gás de aterro (TAO et al., 2008), (CHEN et al., 2008), (HORNG et al., 2009). Assim a produção de hidrogênio como fonte energética e a produção de negro de carbono como insumo industrial se torna 
atrativa e compensa o gasto energético e operacional do sistema (CHEN et al., 2008), (CHAUBEY et al., 2013).

Do ponto de vista ambiental a utilização do plasma é ainda mais vantajosa, pois a partir de fontes poluentes podem ser produzidos produtos com valor agregado e principalmente contribuir com o controle da emissão de gases (BO et al., 2008), (CHEN et al., 2008), (HORNG et al., 2009).

O principal objetivo do presente trabalho foi investigar a eficiência de degradação de metano por plasma térmico, visando a produção de hidrogênio e negro de carbono. Para tanto, foi utilizado um sistema de plasma térmico com tocha de arco não transferido. Os produtos formados, gasosos e sólidos foram identificados e caracterizados por técnicas analíticas.

\section{Materiais e métodos}

\section{Sistema de plasma térmico}

O sistema de plasma térmico utilizado neste trabalho é composto de uma tocha de plasma de arco não transferido (figura 1) e um reator ou câmara de pirólise, projetado para reter ao máximo o fluxo de calor proveniente do jato de plasma. O argônio (Ar) usado como gás de plasma foi introduzido na tocha com vazão controlada através de um rotâmetro (BALDISSARELLI, 2012), (INSTITUTO NACIONAL DA PROPRIEDADE INDUSTRIAL - INPI, 2007), (KHALAF, 2009).
Figura 1 - Tocha de plasma térmico.

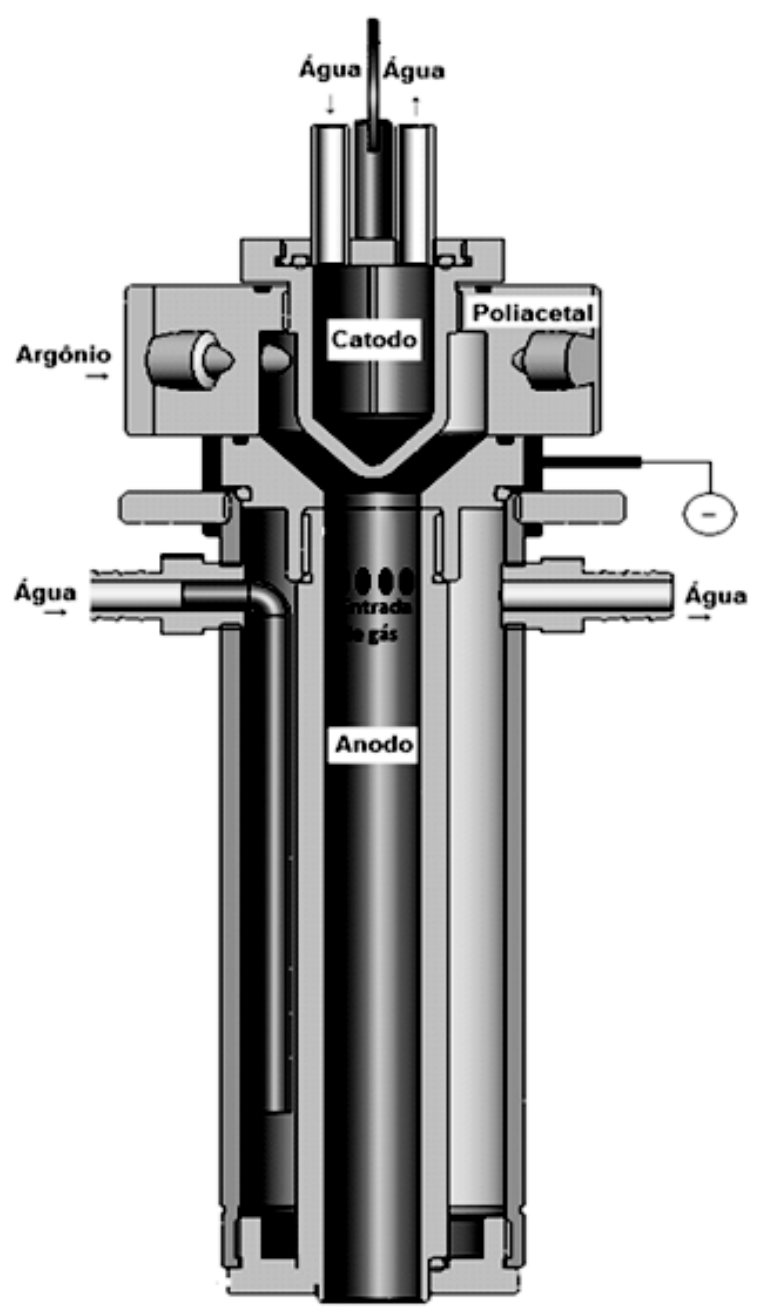

Fonte: (BALDISSARELLI, 2012; INPI， 2007; KHALAF, 2009).

$\mathrm{O}$ arco elétrico entre o cátodo e ânodo foi iniciado por uma descarga de alta tensão. A fonte de alimentação usada foi um conversor CA/CC WEG, modelo CTW - 02.106/3.4 - V2, com entrada de $380 \mathrm{~V}$ (trifásica) e saída de tensão e corrente de até $460 \mathrm{~V}$. A corrente pode chegar até $106 \mathrm{~A}$. A formação do jato de plasma ocorre entre o cátodo e o ânodo (refrigerados por água para evitar sua erosão). O gás a ser degradado $\mathrm{CH}_{4}$ foi introduzido no reator ou câmara de pirólise pela parte inferior, sob fluxo controlado (BALDISSARELLI, 2012), (INPI, 2007), (KHALAF, 2009). 
Detalhes da tocha de plasma são visualizados na figura 1. A tocha consiste de um cátodo de cobre (emissor de elétrons) em forma cônica e um ânodo, também de cobre, em forma de bocal ou canal. O argônio responsável pela formação do plasma é introduzido na tocha através de uma abertura existente no isolante de poliacetal.

O arco elétrico estabelecido entre os eletrodos (cátodo e ânodo) foi iniciado por uma descarga de alta tensão. O gás é aquecido, ionizado e emerge da tocha como um jato de plasma. O jato de plasma formado pode ser observado na figura 2 .

Figura 2 - Tocha de plasma térmico com o jato de plasma

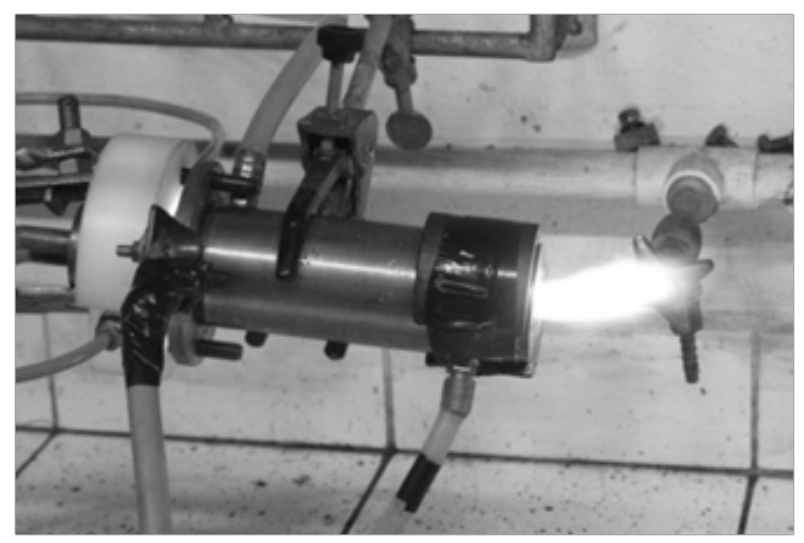

Fonte: (BALDISSARELLI, 2012; INPI, 2007; KHALAF, 2009)

A tocha de plasma foi acoplada à parte superior do reator de plasma, como mostrado na figura 3. O $\mathrm{CH}_{4}$, injetado na parte inferior do reator, foi aquecido e passou, então, para dentro do liner, entrando em contato com o plasma e sofrendo degradação.
Figura 3 - Sistema de plasma acoplada ao reator.

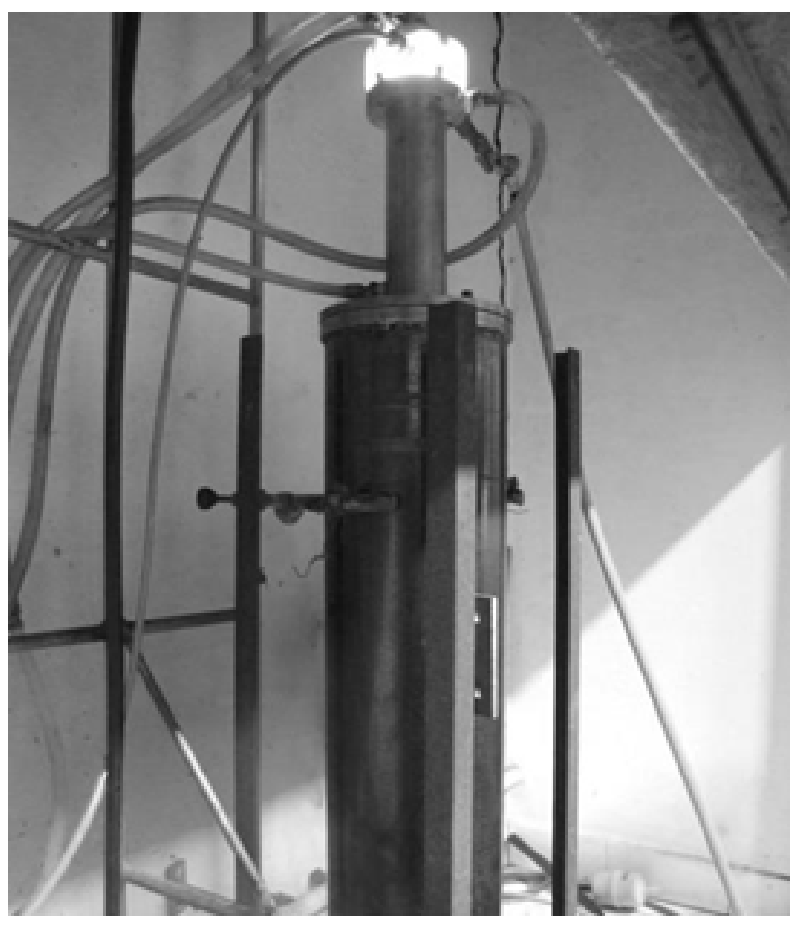

Fonte: (BALDISSARELLI, 2012; INPI， 2007; KHALAF, 2009)

\section{Degradação do metano}

A vazão de argônio foi mantida constante em $20 \mathrm{~L} \mathrm{~min}^{-1}$, e as vazões de $\mathrm{CH}_{4}$ testadas foram 1 , 3, 5 e $7 \mathrm{~L} \mathrm{~min}^{-1}$. A potência aplicada no sistema de plasma foi de $6,8 \mathrm{~kW}$. Todos os gases utilizados neste trabalho foram fornecidos pela White Martins Gases Industriais Ltda.

A morfologia do negro de carbono foi investigada através de microscopia eletrônica de varredura por emissão de campo (FE-SEM) acoplado a um equipamento de espectroscopia de energia dispersiva (EDS) que possibilitou a análise dos elementos químicos presentes na superfície do material.

Amostras gasosas foram coletadas em um amostrador para gases e analisadas através de cromatografia gasosa (modelo Varian 450-GC).

Os brancos foram coletados antes de cada experimento, sendo que os gases passaram pelo 
sistema de plasma com a tocha desligada, na mesma vazão utilizada posteriormente com a tocha ligada. Desse modo, foi possível calcular o número de moles de cada gás presente no sistema antes da degradação, o que equivale ao número de moles de gás introduzido no sistema. Novas coletas foram feitas com o sistema ligado, desse modo foi possível determinar tanto o número de moles de gás não degradado e degradado bem como de outros gases formados no processo.

As análises foram realizadas seguindo uma rampa de aquecimento, com temperatura inicial de $50{ }^{\circ} \mathrm{C}$ por 10 minutos, seguido de taxa de aquecimento de $8{ }^{\circ} \mathrm{C} \min ^{-1}$ até $180{ }^{\circ} \mathrm{C}$, e então mantida essa temperatura por 5 minutos. A temperatura dos detectores foi de $175^{\circ} \mathrm{C}$.

Todas as amostras foram coletadas e analisadas em triplicata. Os valores finais utilizados são as médias destas medidas.

Os produtos da degradação do metano foram identificados por comparação com padrão externo utilizado na calibração do equipamento. Os produtos não identificados representam os hidrocarbonetos formados em pequena escala e os $\mathrm{C}_{6}{ }^{+}$representam os hidrocarbonetos com mais de 6 átomos de carbono.

\section{Cálculos}

A porcentagem de degradação de $\mathrm{CH}_{4}$ foi calculada a partir da equação 1 (TAO et al., 2008), (PIAVIS; TURN, 2012).

$$
\mathrm{X}_{\mathrm{CH} 4}=\left[\left(\mathrm{CH}_{4} \text { cons }\right) /\left(\mathrm{CH}_{4} \text { intro }\right)\right] \times 100 \%
$$

Onde, $X_{\mathrm{CH} 4}$ corresponde à porcentagem de $\mathrm{CH}_{4}$ degradado, $\mathrm{CH}_{4}$ cons corresponde ao número de moles consumido de $\mathrm{CH}_{4}$ e $\mathrm{CH}_{4}$ intro é o número de moles introduzido de $\mathrm{CH}_{4}$. $\mathrm{O}$ número de moles de $\mathrm{CH}_{4}$ consumido foi determinado a partir dos dados cromatográficos obtidos das amostras e o número de moles de $\mathrm{CH}_{4}$ introduzido foi determinado a partir dos dados cromatográficos obtidos dos brancos (TAO et al., 2008), (PIAVIS; TURN, 2012).
$\mathrm{O}$ rendimento na produção de $\mathrm{H}_{2}$ foi calculado através da equação 2 :

$$
\mathrm{X}_{\mathrm{H} 2}=\left[\left(\mathrm{H}_{2} \text { prod }\right) / 2 \mathrm{x}\left(\mathrm{CH}_{4} \text { intro }\right)\right] \times 100 \%
$$

Onde, $X_{H_{2}}$ corresponde à porcentagem de $\mathrm{H}_{2}$ produzido, $H_{2}$ prod é o número de moles produzido, $\mathrm{CH}_{4}$ intro é o número de moles de $\mathrm{CH}_{4}$ introduzido (TAO et al., 2008), (PIAVIS; TURN, 2012).

A partir dos valores obtidos para o rendimento de $\mathrm{H}_{2}$ correspondente a cada uma das vazões de $\mathrm{CH}_{4}$ utilizadas, foi possível calcular a proporção dos subprodutos gasosos formados.

\section{Resultados e Discussões}

Degradação de metano e formação dos produtos

As porcentagens de degradação do $\mathrm{CH}_{4}$ e o rendimento de $\mathrm{H}_{2}$ em diferentes vazões de $\mathrm{CH}_{4}$, mantendo-se constante a vazão de argônio em 20 L $\min ^{-1}$, são mostradas na figura 4. Importante notar que a porcentagem de $\mathrm{CH}_{4}$ convertido diz respeito a quantidade total de $\mathrm{CH}_{4}$ que foi transformado em produtos sólidos e gasosos. Assim, quando a vazão de metano foi ajustada em $1 \mathrm{~L} \mathrm{~min}^{-1}, 59,4 \%$ do gás foi transformado em produtos sólidos e o restante, $40,3 \%$, se refere aos produtos gasosos formados.

$\mathrm{Na}$ figura 4 nota-se que a porcentagem de conversão de $\mathrm{CH}_{4}$ aumenta com o aumento da vazão, chegando a um valor máximo de 98,8 \% na vazão de $5 \mathrm{~L} \mathrm{~min}^{-1}$. Após este máximo, a porcentagem de conversão decai para 94,2 \% em $7 \mathrm{~L} \mathrm{~min}^{-1}$. 
Figura 4 - Porcentagens de conversão do $\mathrm{CH}_{4}(\boldsymbol{\square})$ e rendimento de $\mathrm{H}_{2}(\boldsymbol{\Delta})$ em diferentes vazões de $\mathrm{CH}_{4}$

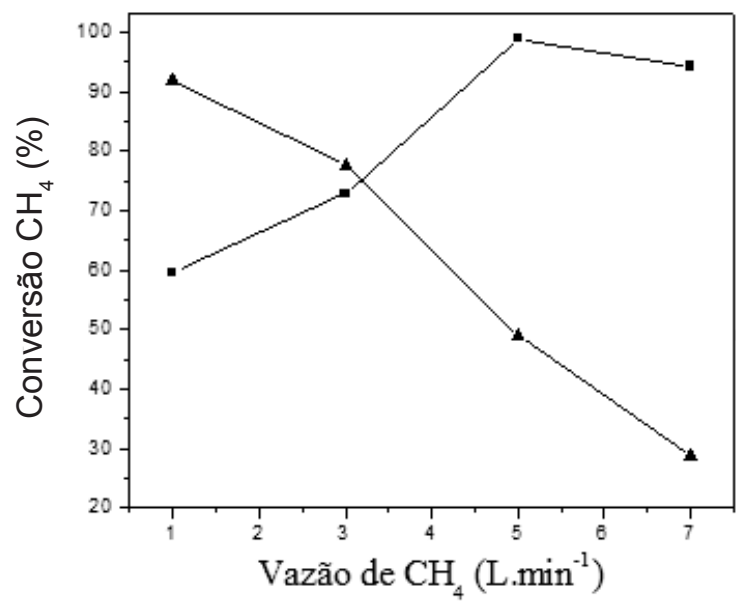

Fonte: Os autores.

A redução proporcional da conversão relacionase a potência elétrica constante aplicada à tocha, uma vez que, a energia disponível é limitada, a conversão de $\mathrm{CH}_{4}$ também será limitada e qualquer quantidade adicional de $\mathrm{CH}_{4}$ permanecerá em excesso no sistema, constituindo a fração não degradada. Uma explicação para a diminuição da conversão é que com o aumento da concentração de $\mathrm{CH}_{4}$, a energia disponível por mol de reagente diminui, uma vez que a potência aplicada na tocha é constante. Para resolver esse problema seria necessário aumentar a potência aplicada e dessa forma permitir que uma maior quantidade de energia fosse aplicada ao sistema, possibilitando assim a conversão de uma quantidade maior de metano (KIM; HONG, 2007). Outra alternativa, seria o aumento da vazão de argônio na tocha de plasma, o que provocaria aumento na temperatura do jato de plasma e consequente aumento da energia do sistema (ABÁNADES; RUBBIA; SALMIERI, 2012).

A decomposição do metano para produção de hidrogênio e carbono sólido é uma alternativa bastante promissora e ambientalmente favorável. Em função da conversão do metano ser endotérmica, a fonte energética (plasma térmico) é bastante eficiente, pois nas condições de plasma, altas temperaturas e elevado grau de ionização, as reações químicas são aceleradas através do fornecimento de energia, além disso, não há emissão de $\mathrm{CO}_{2}$ ou outros gases contaminantes, uma vez que o sistema não é oxigenado (BROMBERG; COHN; RABINOVICH, 1998).

De acordo com Ahmed e colaboradores o processo de degradação de hidrocarbonetos, como o metano, por plasma térmico em fluxos adequados pode produzir até $95 \%$ de $\mathrm{H}_{2}$, o que está coerente com os dados experimentais obtidos neste trabalho (AHMED et al., 2009).

Putra e colaboradores obtiveram uma porcentagem de degradação de $40 \%$ de $\mathrm{CH}_{4}$ e seletividade de $97 \%$ de $\mathrm{H}_{2}$, porém utilizando um sistema de plasma por rádio frequência (PUTRA et al., 2012).

Taghvaei e colaboradores mostraram que com o aumento da vazão de metano para um valor fixo de vazão de argônio, o número de produtos gasosos (etileno, propileno, acetileno, etc) aumentam, o que leva a uma diminuição da produção de $\mathrm{H}_{2}$. (TAGHVAEI et al., 2013).

A degradação do $\mathrm{CH}_{4}$ envolve várias etapas reacionais. Muitos estudos já foram realizados com o objetivo de descrever estas etapas e propor um mecanismo geral. Um dos mecanismos mais aceitos foi proposto por Kassel e é determinado pela formação de radicais $\mathrm{CH}_{2}$ (KASSEL 1932 apud FRIDMAN, 2008, p. 589).

$$
\mathrm{CH}_{4} \rightarrow \mathrm{CH}_{2}+\mathrm{H}_{2}
$$

$\mathrm{O}$ radical $\mathrm{CH}_{3}$ também contibui para a reação, mas é formado em menor extensão do que do $\mathrm{CH}_{2}$. Depois da etapa de formação dos radicais $\mathrm{CH}_{2}$, estes podem levar a conversão de metano a etano, como mostrado a seguir.

$$
\mathrm{CH}_{4}+\mathrm{CH}_{2} \rightarrow \mathrm{C}_{2} \mathrm{H}_{6}
$$


Etapas adicionais levam, gradualmente, à formação de etileno a partir do etano, depois de acetileno a partir de etileno e finalmente do negro de carbono, conforme equações abaixo:

$$
\begin{aligned}
& \mathrm{C}_{2} \mathrm{H}_{6} \rightarrow \mathrm{C}_{2} \mathrm{H}_{4}+\mathrm{H}_{2} \\
& \mathrm{C}_{2} \mathrm{H}_{4} \rightarrow \mathrm{C}_{2} \mathrm{H}_{2}+\mathrm{H}_{2} \\
& \mathrm{C}_{2} \mathrm{H}_{2} \rightarrow 2 \mathrm{C}_{(\mathrm{S})}+\mathrm{H}_{2}
\end{aligned}
$$

Também foram detectados outros produtos gasosos em menor escala, como por exemplo, $\mathrm{C}_{6}+$ (hidrocarbonetos com mais de 6 carbonos), butadieno, butino, propino, entre outros. Considerando que o $\mathrm{CH}_{4}$ utilizado nos testes tem uma concentração de $99 \%$, impurezas também podem gerar reações paralelas isso justifica os resultados encontrados (KASSEL 1932 apud FRIDMAN, 2008, p. 589), (KHALAF et al., 2011).

\section{Produtos gasosos formados}

A partir dos dados obtidos das análises de CG foi possível identificar produtos gasosos formados em menor escala e estimar a porcentagem de formação de alguns deles.

Tabela 1 - Produtos gasosos formados pela degradação de $\mathrm{CH}_{4}$ por plasma térmico.

\begin{tabular}{ccccc}
\hline \multirow{2}{*}{ Compostos } & \multicolumn{4}{c}{ Vazão de $\mathrm{CH}_{4}\left(\mathrm{~L} \mathrm{\text {min}^{-1 } )}\right.$} \\
& 1 & 3 & 5 & 7 \\
\cline { 2 - 5 } & \multicolumn{4}{c}{ Produtos formados (\%) } \\
\hline $\mathrm{H}_{2}$ & 91,9 & 77,7 & 48,9 & 28,8 \\
$\mathrm{CH}_{4}$ (saída) & 0,2 & 7,8 & 1,9 & 9,9 \\
Acetileno & 6,2 & 11,1 & 33,0 & 39,8 \\
Etileno & 0,4 & 1,8 & 11,9 & 13,0 \\
* $_{6}+$ & 0,2 & 0,4 & 1,0 & 4,7 \\
Etano & 0,0 & 0.0 & 0,4 & 0,3 \\
Butadieno & 0,0 & 0,0 & 0,4 & 0,4 \\
Butino & 0,1 & 0,2 & 0,6 & 1,2 \\
Propino & 0,0 & 0,0 & 0,0 & 0,1 \\
Não identificados & 1,0 & 1,0 & 1,9 & 1,9 \\
\hline Total & \multicolumn{5}{c}{100} \\
\hline
\end{tabular}

Fonte: Os autores.
$\mathrm{Na}$ tabela 1 os dados de porcentagens dos produtos sólidos formados foram desconsiderados e os dados na tabela se restringem as proporções formadas apenas para os produtos gasosos. Assim, considera-se que as porcentagens expostas na tabela 1 dizem respeito apenas aos produtos gasosos obtidos em cada ajuste de vazão. Por exemplo, na vazão de $1 \mathrm{~L} \mathrm{~min}^{-1}$, 40,3\% de produtos gasosos foram formados, como pode ser observado na figura

4. Para as outras vazões os valores que se referem ao total de produtos gasosos são: $3 \mathrm{~L} \mathrm{~min}^{-1}, 27,1 \%$ de produtos gasosos; $5 \mathrm{~L} \mathrm{~min}^{-1}, 1,1 \%$ de produtos gasosos; $7 \mathrm{~L} \mathrm{~min}{ }^{-1}, 5,7 \%$ de produtos gasosos. Assim, o total de $100 \%$ exposto na tabela 1 é proporcional apenas aos produtos gasosos formados em cada vazão, excluindo-se as quantidades de material sólido formado e de metano que não foi convertido.

De acordo com a tabela 1 , pode ser verificado que quanto menor a vazão de $\mathrm{CH}_{4}$, maior a porcentagem de produtos gasosos formados. O hidrogênio é o principal produto formado em vazões abaixo de 7 $\mathrm{L}$ min $^{-1}$ enquanto o acetileno é o principal produto em vazões elevadas $\left(7 \mathrm{~L} \mathrm{~min}^{-1} \mathrm{de} \mathrm{CH}_{4}\right)$. A proporção de etileno e de $\mathrm{C}_{6}+$ na mistura obtida também é considerável, chegando a $12,9 \%$ e 4,6 \% (a 7 L min ${ }^{1} \mathrm{de}_{\mathrm{CH}_{4}}$ ), respectivamente.

Segundo Fincke e colaboradores em fluxos de $\mathrm{CH}_{4}$ mais baixos (entre 1 a $5 \mathrm{~L} \mathrm{~min}^{-1}$ ), onde teoricamente os produtos de equilíbrio ficam em temperaturas entre 1000 e $2500{ }^{\circ} \mathrm{C}$, os principais produtos formados na degradação do $\mathrm{CH}_{4}$ são o negro de carbono e o $\mathrm{H}_{2}$ (FINCKE et al., 2002a).

Considerando o mecanismo de Kassel, descrito anteriormente, equações 3 a 7, podemos assumir que a formação de acetileno e $\mathrm{CH}_{4}$ não degradado no sistema vão aumentando em vazões maiores de $\mathrm{CH}_{4}$ porque a energia aplicada é constante e insuficiente para degradar todo o $\mathrm{CH}_{4}$ até as etapas finais, formando assim subprodutos em maiores proporções (KASSEL 1932 apud FRIDMAN, 2008, p. 589). 
Outra hipótese é que com o aumento dos produtos de reação, pode ocorrer o resfriamento rápido destes gases em temperaturas em que são estáveis, antes do processo da formação, nucleação e crescimento do carbono amorfo. Como resultado, a composição do equilíbrio não é atingida e o acetileno e outros subprodutos gasosos são formados em maior escala (BALDISSARELLI, 2012), (FINCKE et al., 2002), (BROMBERG; COHN; RABINOVICH, 1998).

Yan e colaboradores mostraram que a temperatura interna do reator é um dos fatores que interfere diretamente nos produtos gasosos formados. O trabalho mostra resultados especialmente em relação à identificação dos produtos gasosos e as diferentes porcentagens de formação destes produtos em diferentes temperaturas de degradação (YAN et al., 2012).

\section{Produto sólido}

A figura 5 apresenta uma imagem obtida por FESEM do carbono sólido. Pode ser observado que sua superfície é formada por agregados de estrutura amorfa e que as partículas têm tamanhos entre 200 e $300 \mathrm{~nm}$ (BALDISSARELLI, 2012), (LIU et al., 2012), (KANG; LI; SAITO, 2013). Os resultados da análise qualitativa de EDS mostraram composição superficial de $100 \%$ de carbono, o que mostra que o material produzido é negro de carbono de alta pureza.

O material apresentou características hidrofóbicas, o que são coerentes com os dados da literatura para o negro de carbono comercial (ONO et al., 2013), (ABÁNADES; RUBBIA; SALMIERI, 2012).
Figura 5 - Imagem de FE-SEM do carbono sólido obtido pela degradação de $\mathrm{CH}_{4}$ por plasma térmico.

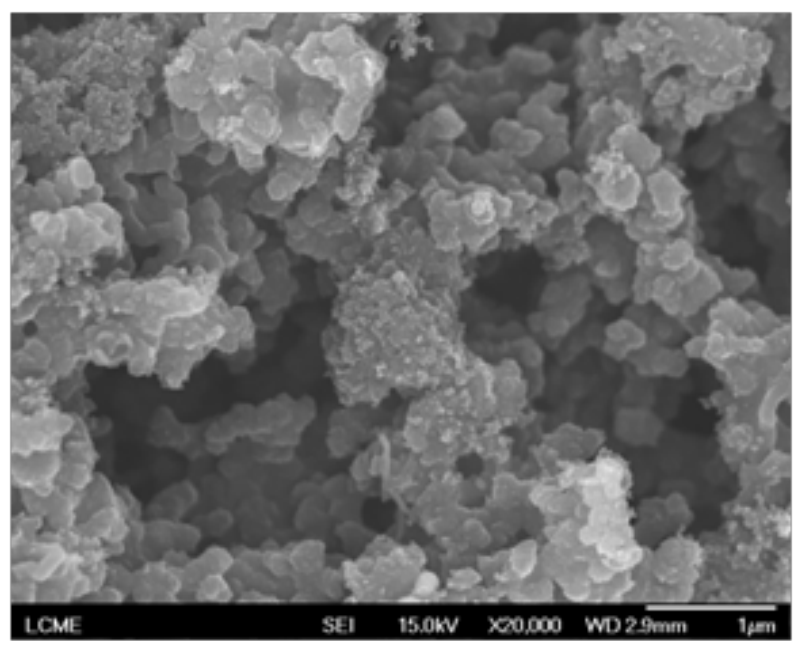

Fonte: Os autores.

De acordo com a literatura, o negro de carbono é um material coloidal na forma de esferas e agregados. É um produto comercial obtido por decomposição térmica ou combustão parcial de hidrocarbonetos e possui uma morfologia bem definida. Em contraste com a fuligem, que contém contaminantes inorgânicos e resíduos orgânicos, negros de carbono comerciais contém cerca de 97 a $99 \%$ de carbono elementar, dependendo do processo de produção (KHALAF, 2009), (ONO et al., 2013).

No trabalho desenvolvido por Kim e colaboradores foi mostrado o efeito da temperatura no tamanho das partículas de negro de carbono produzidas. Com o aumento da potência aplicada e, consequentemente, da temperatura do plasma, houve um aumento do tamanho das partículas produzidas. Em potências menores, os produtos consistem de agregados de pequenas partículas de carbono (KIM; HONG, 2007).

Resultados similares foram obtidos no trabalho desenvolvido por Fincke e colaboradores, onde foi investigada a formação de negro de carbono a partir da pirólise de diferentes substâncias, entre elas: etileno, metano, estireno e óleo combustível (FINCKE et al., 2002b). 


\section{Conclusões}

A pirólise de $\mathrm{CH}_{4}$ por plasma térmico mostrouse eficiente, com bons rendimentos de negro de carbono e $\mathrm{H}_{2}$, e com vantagens em relação a outras técnicas, pois o processo não libera $\mathrm{CO}_{2}$.

A porcentagem de degradação do $\mathrm{CH}_{4}(5 \mathrm{~L}$ $\min ^{-1}$ ) chega ao valor máximo de $98,8 \%$ para uma potência aplicada de $6,8 \mathrm{~kW}$.

A formação de $\mathrm{H}_{2}$ é favorecida em vazões menores de $\mathrm{CH}_{4}$. Com o aumento da vazão, a formação de outros produtos gasosos como o acetileno é favorecida. As porcentagens de $\mathrm{H}_{2}$ chegaram a 91,9 \% e de acetileno a $39,7 \%$ quando a vazão de $\mathrm{CH}_{4}$ foi ajustada em 1 e $7 \mathrm{~L} \mathrm{~min}^{-1}$, respectivamente.

Outros produtos gasosos foram produzidos em diferentes proporções durante o processo, destacando-se, o acetileno, etileno e os $\mathrm{C}_{6}+$.

O negro de carbono produzido através da degradação do $\mathrm{CH}_{4}$ em plasma térmico tem características hidrofóbicas, é amorfo e de alta pureza, apresentando valor agregado e podendo ser comercializado.

\section{Agradecimentos}

Os autores agradecem o apoio financeiro da Capes, CNPQ, Universidade Federal de Santa Catarina, Carbono Brasil Ltda, LCME-UFSC pelas microscopias e INCT-Catálise pelas análises químicas.

\section{Referências}

ABÁNADES, A.; RUBBIA, C; SALMIERI, D. Technological challenges for industrial development of hydrogen production based on methane cracking. Energy, Matsuyama, v. 46, p. 359-363, 2012.

AHMED, S.; AITANI, A.; RAHMAN, F.; ALDAWOOD, ALI.; AL-MUHAISH, F. Decomposition of hydrocarbons to hydrogen and carbon. Applied Catalysis A: general, Karachi, v. 359, p. 1-24, 2009.
BALDISSARELLI, V. Z. Estudo da degradação do $\mathrm{CH}_{4}$ e da mistura $\mathrm{CH}_{4} \mathrm{CO}_{2}$ por plasma térmico de Argônio: Identificação e caracterização dos produtos obtidos. 2012. Tese (Doutorado em Química) - Universidade Federal de Santa Catarina - UFSC, Florianópolis. Disponível em: $\quad<$ http://aspro02.npd.ufsc.br/pergamum/ biblioteca/index.php?resolution2=1024_1\&tipo_ pesquisa $=\#$ posicao_dados_acervo $>$. Acesso em: 17 de junho de 2013.

BENETOLI, L. O.; CADORIN, B. M.; BALDISSARELLI, V. Z.; GEREMIAS, R.; SOUZA, I. G.; DEBACHER, N. A. Pyrite-enhanced methylene blue degradation in non-thermal plasma water treatment reactor. Journal of Hazaurdous Materials, Florianopolis, v. 237-238, p. 55-62, 2012.

BO, Z.; YAN, J.; LI, X.; CHI, Y.; CEN, K. Plasma assited dry methane reforming using gliding arc gas discharge: Effect of feed gases proportion. International Journal of Hydrogen Energy, Zhejiang, v. 33, p. 5545-5553, 2008.

BOULOS, M. I.; FAUCHAIS, P.; PFENDER, E. Thermal plasma. New York: Plenum press, 1994. v. 1.

BROMBERG, L.; COHN, D. R.; RABINOVICH, A. Plasma Reforming of Methane. Energy \& Fuels, v. 12, p. 11-18, 1998.

CHAUBEY, R.; SAHU, S.; JAMES, O. O.; MAITY, $\mathrm{S}$. A review on development of industrial processes and emerging techniques for production of hydrogen from renewable and sustainable sources. Renewable and Sustainable Energy Reviews, Dhanbad, v. 23, p. 443-462, 2013.

CHEN, H. L.; LEE, H. M.; CHEN, S. H.; CHAO, Y.; CHANG, M. B. Review of plasma catalysis on hydrocarbon reforming for hydrogen production - Interaction, integration, and prospects. Applied Catalysis B: environmental, Taoyuan, v. 85, p. 1-9, 2008. 
CUBAS, A. L. V.; CARASECK, E.; DEBACHER, N. A.; SOUZA, I. G. Development of a DC-Plasma torch constructed with graphite electrodes and an integrated nebulization system for decomposition of $\mathrm{CCl}_{4}$. Journal of Brazilian Chemical Society, Florianopolis, v. 16, n. 3, p.531-534, 2005.

EMMERT, S.; BREHMER, F.; HANBLE, H.; HELMKE, A.; MERTENS, N.; AHMED, R.; SIMON, D.; WANDKE, D.; MAUS-FRIEDRICHS, W.; DASCHLEIN, G.; SCHON, M. P.; VIOL, W. Atmospheric pressure plasma in dermatology: Ulcus treatment and much more. Clinical Plasma Medicine, Berlin, v. 1, p. 24-29, 2013.

FABRY, F.; FLAMANT, G.; FULCHERI, L. Carbon black processing by thermal plasma - Analysis of the particle formation mechanism. Chemical Engineering Science, Salford, v. 56, p. 2123-2132, 2001.

FARRELL， P.; CULLING, D.; LEIFER, I. Transcontinental methane measurements: Part 1. A mobile surface platform for source investigations. Atmospheric Environment, Arhus v. 74, p. 422-431, 2013.

FINCKE, J. R.; ANDERSON, R. P.; HYDE, T.; DETERING, B. A.; WRIGHT, R.; BEWLEY, R. L.; HAGGARD, D. C.; SWANK, W. D. Plasma thermal conversion of methane to acetylene. Plasma Chemistry and Plasma Processing, Boston, v. 22, p. 105-136, 2002a.

FINCKE, J. R.; ANDERSON, R. P.; HYDE, T. A.; DETERING, B. A. Plasma pyrolysis of methane to hydrogen and carbon black. Industrial \& Engineering Chemistry Research, Boston, v. 41, p. 1425-1435, 2002b.

FRIDMAN, A. Plasma chemistry. New York: Cambridge University Press, 2008.

HORNG, R F.; LAI, M. P.; CHANG, Y. P.; YUR, J P.; HSIEH, S. F. Plasma-assited catalytic reforming of propane and an assessment of its applicability on vehicles. International Journal of Hydrogen Energy, Tainan, v. 34, p. 6280-6289, 2009.
KANG, J.; LI, O. L.; SAITO, N. Syntheis of structurecontrolled carbon nano spheres by solution plasma process. Carbon, Nagoya, v. 60, p. 292-298, 2013.

GUO, X. F.; KIM, G. J. Ultrafine carbon black produced by pyrolysis of polyethylene using a novel DC-thermal plasma process. Journal of Physics and Chemistry of Solids, Seoul, v. 69, p. 1224-1227, 2008.

KHALAF, P. I. Obtenção de hidrogênio, carbono sólido e gás de sintese por plasma térmico de argônio a partir da degradação de metano, biogás e água. 2009. Tese (Doutorado em Química) - Universidade Federal de Santa Catarina - UFSC, Florianópolis. Disponível em: $\quad<$ http://aspro02.npd.ufsc.br/pergamum/ biblioteca/index.php?resolution2=1024_1\&tipo_ pesquisa=\#posicao_dados_acervo $>$ Acesso em: 20 jun. 2013.

KHALAF, P. I.; SOUZA, I. G.; CARASEK, E.; DEBACHER, N.A. Produção de gás de síntese por plasma térmico via pirólise de metano e dióxido de carbono. Química Nova, Florianopolis, v. 34, n. 9, p. 1491-1495, 2011.

KIM, K. S.; SEO, J. H.; NAM, J. S.; JU, W. T.; HONG, S. H. Production of hydrogen and carbono black by methane decomposition using DC-RF hybrid thermal plasmas. IEEE Transactions on Plasma Science, Seoul, v. 33, p. 813-823, 2005.

KIM, K. S.; HONG, S. H; Continuous Synthesis of nanostructured sheetlike carbons by termal plasma decomposition of methane. IEEE Transactions on Plasma Science, Seoul, v. 35, p. 434-443, 2007.

LIU, B.; HUANG, H.; ZHANG, F.; ZHOU, Y.; LI, W.; ZHANG, J. Agglomerates of amorphous carbon nanoparticles synthesized by a solution-phase method. Materials Letters, Shanghai, v. 66, p. 199-202, 2012.

INSTITUTO NACIONAL DA PROPRIEDADE INDUSTRIAL - INPI: Processo de degradação de gases causadores do efeito estufa e retenção do material particulado. Tocha de plasma e reator de plasma. 2007. PI-0700517-2 de 15/02/2007 e PCTBR2007/000116 de 15/05/2007; WO 2008/098324 A1, 21/08/08. 
ONO, K.; YANAKA, M.; SAITO, Y.; AOKI, H.; FUKUDA, O.; AOKI, T. Effect of benzene-acetylene compositions on carbon black configurations produced by benzene pyrolysis. Chemical Engineering Journal, Myagi, v. 215, p. 128-135, 2013.

SAMAL, S.; PARK, D-W. Nano-particle synthesis of titanium oxides from ilmenite in a thermal plasma reactor. Chemical Engineering Research and Design, Incheon, v. 90, p. 548-554, 2012.

STAACK, D. E. A. DC normal glow discharges in atmospheric pressure atomic and molecular gases. Plasma Sources Science and Technology, Homburg, v. 17, p. 13-25, 2008.

PIAVIS, W.; TURN, S. An experimental investigation of reverse vortex flow plasma reforming of methane. International Journal of Hydrogen energy, v. 37, p. 17078-17092, 2012.

PUTRA, A. E. E.; NOMURA, S.; MUKASA, S.; TOYOTA, H. Hydrogen production by radio frequency plasma stimulation in methane hydrate at atmospheric pressure. International Journal of Hydrogen Energy, Matsuyama, v. 37, p. 6000-6005, 2012.

REDDY, P. V. L.; KIM, K-H.; SONG, H. Emerging green chemical technologies for the conversion of $\mathrm{CH} 4$ to value added products. Renewable and Sustainable Energy Reviews, Seoul, v. 24, p. 578585, 2013.

TAGHVAEI, H.; JAHANMIRI, A.; RAHIMPOUR, M. R.; SHIRAZI, M. M.; HOOSHMAND, N. Hydrogen production through plasma cracking of hydrocarbons: Effect of carrier gas and hydrocarbon type. Chemical Engineering Journal, Shiraz, v. 226, p. 384-392, 2013.

TAO, X.; QI, F.; YIN Y.; DAI, X. $\mathrm{CO}_{2}$ Reforming of $\mathrm{CH}_{4}$ by Combination of Thermal Plasma and Catalyst. International Journal of Hydrogen Energy, v. 33, p. 1262-1265, 2008.
XU, Y.; WEI, Q.; LONG, H.; ZHANG, X.; SHANG, S.; DAI, X.; YIN, Y. $\mathrm{CO}_{2}$ rerforming of $\mathrm{CH}_{4}$ by synergies of binode thermal plasma and catalysts. International Journal of Hydrogen Energy, Liverpool, v. 38, p. 1384-1390, 2013.

YAN, B.; CHENG, Y.; JIN, Y.; GUO, C. Y. Analysis of particle heating and devolatilization rapid coal pyrolysis in thermal plasma reactor. Fuel Processing Technology, Beijing, v. 100, p. 1-10, 2012.

Recebido em 23 Julho 2013-Received on July 27, 2013. Aceito em 17 Março, 2014 - Accepted on March 17, 2014. 
